\title{
ANÁLISIS Y SIMULACIONES EN BASE A LAS MATRICES INSUMO-PRODUCTO DE LA COMUNA DE PUNTA ARENAS PARA LOS AÑOS 2003 Y $2007^{1}$
}

\author{
SERGIO SOZA-AMIGO* Y PATRICIO AROCA*
}

\begin{abstract}
RESUMEN
Una matriz de insumo-producto es como una radiografía del sistema productivo de una economía que resume los fundamentos y la actividad económica ocurrida durante un año. Soza-Amigo et al en 2010 y 2012, estimaron las matrices de insumo-producto de la Comuna de Punta Arenas para los años 2003 y 2007. Basados en estas estimaciones, este trabajo presenta las principales características económicas de la comuna para ambos años y los principales cambios estructurales ocurridos en el quinquenio. Los resultados indican que aún cuando hay actividades basadas en la explotación de materias primas con una alta participación en el PIB, existe un conjunto de servicios que esta ganando en importancia y en impactos. Adicionalmente, los encadenamientos en general han crecido entre los dos periodos, sin embargo, los cambios en los multiplicadores de producto han sido mucho más significativos que los ocurridos en los ingresos, implicando que probablemente los mayores niveles de producción están asociados a incrementos significativos de capital en la economía de Punta Arenas.
\end{abstract}

PALABRAS CLAVE: matrices insumo-producto, cambio estructural, simulación.

Clasificación JEL: C67; D57; R15; R34 y Z00.

1 Se reconocen y agradecen los financiamientos entregados por FONDECYT; por medio de los proyectos de investigación "Evaluación Económica de la Política Regional en Chile", Código 1111061 y, "Entre el Conflicto y el Aislamiento: Desarrollo, Gobernabilidad y Seguridad en las Zonas Fronterizas del Norte y el Sur de Chile a Comienzos del Siglo XXI", Código: 1120405; asi como, a la ayuda recibida por parte del Ministerio de Economía, Fomento y Turismo, Proyecto NS 100046, de la Iniciativa Científica Milenio y también por su Red Insumo- Producto y, finalmente al Gobierno Regional de Magallanes y Antártica Chilena, por medio del Proyecto FIC-2010 "Cambios Estructurales y el Efecto del Turismo en la Economía de Punta Arenas", Código BIP: 30106900-0.

* Facultad de Ciencias Económicas y Jurídicas, Universidad de Magallanes, Punta Arenas, Chile, e-mail: sergio.soza@umag.cl.

* Director del Instituto de Economía Aplicada Regional (IDEAR), Universidad Católica del Norte, Antofagasta, Chile, e-mail: paroca@ucn.cl. 


\section{ANALYSIS AND SIMULATIONS BASED ON INPUT - OUTPUT MATRIX OF PUNTA ARENAS DISTRICT FOR YEARS 2003 AND 2007.}

\section{ABSTRACT}

An input-output matrix is like an x-ray of the productive system of an economy that shows the health of the fundamentals and survey the economic activity developed during a year. Soza-Amigo et al (2010 and 2012) estimated Punta Arenas county input-output matrices for the years 2003 and 2007. Based on that estimation, this paper presents the economic characteristics of the county for both years and the main structural changes occurred during the five years under study. The results show that while some primary activities are very important on the regional gross domestic product, they are a set of services that is increasing their share in the production. Also a significant increment in the output multipliers is taking place; the change in multipliers has been significantly lower than those occurred in income, which is suggesting that the changes might be associated to significant capital increase in Punta Arenas economy.

KEYWORDS: input-output matrix, structural change, simulations.

Classification JEL: C67; D57; R15; R34 y Z00.

\section{INTRODUCCIÓN}

Utilizando las matrices de insumo-producto estimadas para Punta Arena para los años 2003 y 2007, se describen la importancia de los principales sectores productivos de la comuna, los cambios ocurridos en el quinquenio tanto en los niveles de producción como en los multiplicadores de producto e ingreso.

Los resultados sugieren que Punta Arenas sigue siendo una economía basada en recursos naturales, con un incremento significativo de los sectores de servicios que primero levantan una nueva incógnita sobre si estos cambios son genuinamente la generación de un nuevo grupo de actividades, probablemente asociadas al sector turismo, o corresponde a una estrategia de subcontratación adoptada por las grandes empresas del sector primario, aspectos que estarían acorde con lo presentado por Soza- Amigo en 2008 y 2009.

También se aprecia un significativo incremento en los multiplicadores de producto, lo que no se traduce en un incremento similar en los multiplicadores de ingreso, lo que hace presumir que los cambios ocurridos han estado principalmente asociados a incrementos en capital.

En la siguiente sección se presentan los principales resultados para un resumen de solo 3 sectores económicos, para en las secciones siguientes expandir este análisis a los 12 principales sectores de los 40 contenidos en las matrices disponibles para los años 2003 y 2007. Finalmente, se presenta un análisis de impacto vía multiplicadores y las conclusiones.

\section{PRINCIPALES RESULTADOS}

Con el fin de ilustrar, explicar y destacar los principales resultados de la Matriz de Insumo-Producto de la Comuna de Punta Arenas elaborada para el año 2007 (MIPPA-07) y proceder posteriormente con su comparación para la existente del año 2003 (MIPPA-03) ${ }^{2}$, se ha procedido con una agregación de la mismas a los tres sectores característicos de una economía: primario (extracción de recursos), secundario (industria) y terciario (servicios) ${ }^{3}$, aún cuando se es consciente que la agregación es alta, ella permite una análisis interesante de la economía que se estudia 4 . Uno de los primeros resultados que se desprenden y destacan de la MIPPA-07 es su PIB, el mismo para el año 2007 era de 804.577 millones de pesos (valorados en pesos del año 2003), lo

2 Para ambos casos las matrices originales viene confeccionadas a 40 actividades y productos.

3 El procedimiento realizado se incluye en anexo 1, 2 y 3, y en el anexo 7 y 8 las matrices para el año 2003 y 2007.

4 Para un mayor detalle de los efectos de la agregación en matrices input-output, se puede revisar SOZA-Amigo, S. y C. RAMOS (2011). 
que implica una reducción de 27.925 millones de pesos respecto del año 2003 (832.502 millones de pesos del año 2003), es decir, de algo más de un $3 \%$; por otro lado, se aprecia que su VBP (valor bruto de producción), se estimo para el año 2007 en 1.826.493 millones de pesos del año 2003, algo más de un 15\% superior al logrado en 2003 (1.587.116 millones de pesos del año 2003).

\section{ANÁLISIS DE LA MATRIZ INSUMO-PRODUCTO 2007}

Una de las características y ventajas importantes de la modelación de tablas de insumo-producto (TIO), es el identificar e ilustrar el intercambio entre las distintas actividades y productos que forman una economía, lo cual se hace por medio de la transformación de relaciones funcionales; esto es, a partir de los distintos requerimientos de insumo de una actividad, en las TIOs se plasma la estructura técnica que permite establecer cuál o cuáles son las relaciones en que se involucra una actividad o producto en términos de uso e intercambio con el resto del sistema económico. En consonancia con lo anterior, lo que continúa responde a identificar esas relaciones, con este fin, se abordará la matriz de coeficientes técnicos y de distribución, poniendo atención a la forma en que se obtienen y a lo que representan sus distintos coeficientes.

Para obtener los coeficientes técnicos, se debe dividir cada uno de los componentes de la matriz de consumos intermedios y los de la matriz de insumos primarios por su correspondiente valor bruto de la producción (VBP).

Matemáticamente, un coeficiente técnico o elemento de la matriz de insumo-producto, se define como: $\mathrm{a}_{\mathrm{ij}}=\mathrm{X}_{\mathrm{ij}} / \mathrm{X}_{\mathrm{i}}$ con $\mathrm{a}_{\mathrm{ij}} \hat{\mathrm{I}} \mathrm{A}$, donde $\mathrm{X}_{\mathrm{j}}$ corresponde Valor Bruto de la Producción del j-ésimo sector, mientras que $\mathrm{X}_{\mathrm{ij}}$, representa los insumos requeridos del i-ésimo sector por el j-ésimo sector.

Cada columna de la matriz de coeficientes técnicos es una aproximación de una "función de producción"; la que representa la receta técnica de cómo elaborar un producto o servicio de una determinada actividad o sector; además, cada matriz tendrá tantas funciones como columnas de transacciones intermedias. Luego, cada coeficiente técnico indica, que proporción de su producción compra a la actividad o producto con que se relaciona.
Para entender lo anterior, se parte de la matriz insumo-producto del año 2007 (Anexo 3) y la de coeficientes técnicos del año 2007 (Anexo 4); y, se tomará como ejemplo, el sector primario (s1); a continuación y tomando como referencia el elemento fila 2 y columna 1 de la matriz insumo-producto $\left(z_{1 ; 2}\right)$, se mostrará cuál es la relación entre el sector extractivo (s1) con el manufacturero (s2), esto es, el coeficiente técnico $\mathrm{a}_{1 ; 2}=0.28=62.010 / 221.533$. Esta cifra indica que, para producir una unidad de producto del sector extractivo, se requiere gastar 0.28 en la industria manufacturera, o lo que podría interpretarse como: del gasto que se realiza para producir el total de producción del sector primario extractivo, incluyendo salarios, ganancias, impuestos e importaciones, un $28 \%$ se hace en compras al sector secundario o manufacturero. La obtención del resto de los coeficientes e interpretación de los mismos es análoga a la ya presentada.

En el caso de la matriz de distribución (Anexo 5), la forma de obtener sus elementos es similar a lo expuesto, pero en vez de dividir en un sentido de columnas se hace por filas. Tomando nuevamente como referencia el sector primario (s1), considere su interacción con el sector terciario (s3), se tendrá que el valor $0.05=109.267 / 221.533$; indica que un $5 \%$ de los productos del sector extractivo se venden al sector terciario (s3).

Centrando el estudio en la composición de la matriz de coeficientes técnicos (efectos directos), se observa; que para el año 2007 el sector primario demandaba una importante proporción del sector secundario, es decir, dependida significativamente de éste último (aproximadamente un 30\%); por otra parte, también se aprecia, que los consumos intermedios son levemente superiores al valor agregado (un 53 versus un 46\% aproximadamente).

Basados en los multiplicadores que se obtienen de la matriz inversa de Leontief (Anexo 6), para una agrupación de tres ramas, el sector industrial es el que genera un mayor impacto sobre el resto de la economía (2,80 en columna y 3,41 en fila), su efecto multiplicador señala que; de producirse un incremento en su demanda final de; por ejemplo, 100 millones de pesos valorados al año 2003, la economía vería incrementada su producción en algo más de 280 millones de pesos $\left(2,80^{*} 100.000 .000\right)$. Separando el impacto por sector, lo anterior implicaría que; 17.140 .000 pesos se requerirían del sector 
primario para satisfacer el incremento de los 100 millones; 41.520 .000 pesos del sector servicios; y, la diferencia, algo más de 220 millones, serían requeridos por la propia actividad industrial, los que se distribuyen en los 100 millones de la demanda final mas requerimientos intermedios para la propia actividad. En línea con lo anterior, pero ahora analizando la suma de las filas de la matriz inversa de Leontief, se observa que es nuevamente en el sector industrial donde se encuentra el mayor multiplicador $(3,41)$, tal valor señala que, si se produce un aumento unitario de la demanda final para el conjunto de la economía; o, en otras palabras, si aumenta la demanda final de una actividad en una unidad monetaria, la economía necesitaría en total de 3,41 unidades monetarias, las que se distribuirían en el siguiente orden; 0,77 del primario, 2,22 del secundario; y, 0,43 del sector servicio.

En una lógica similar a la anterior pero, para la matriz inversa de distribución o de Ghosh (Anexo 7), se observa que tanto en filas como en columnas, el mayor valor se da nuevamente para el sector manufacturero (secundario; 2,87 y 3,24 respectivamente). El primer valor señala que (multiplicador de oferta o de inputs); si se produce un aumento de una unidad en los insumos primarios (excedente bruto de explotación, remuneraciones e impuestos netos) de tal actividad, el sistema vería incrementada su producción total en 2,87 unidades monetarias, distribuyéndose ellas como sigue: 0,21 en el sector primario; 2,22 en el industrial y; 0,44 en el sector servicios. Por su parte, el valor en columna señala que, de existir una baja de una unidad monetaria en los insumos primarios del sector en si -independientemente del en que, entiéndase ello por; excedente bruto de explotación, salarios o impuestos netos-, la producción se vería reducida; en general, en 3,24 unidades monetarias, esto es; 0,61 unidades producto de su relación con el sector primario; 2,22 de propia relación de autoconsumo; $y$, en 0,41 unidades de su vínculo con el sector servicios.

Una mirada amplia de los multiplicadores de producción (suma en columnas); los cuales responden al "en cuánto" se debe incrementar la producción de todo el sistema económico con el fin de satisfacer los requerimientos solicitados al mismo para poder cumplir con el incremento de una unidad en la demanda final de un sector específico; $y$, por su parte, para los multiplicadores de oferta o de insumos (suma en filas de la matriz inversa de Ghosh); los cuales señalan "por cada actividad" cuál es el aporte que la misma debe realizar con el fin de que aumenten en una unidad los insumos primarios. En ambos casos (demanda y oferta), los multiplicadores indican que; en general, en términos de requerimientos causados por una expansión de la demanda final, afectan más sobre el sistema, las actividades y productos ligados a la extracción e industria manufacturera, lo que se ha detectado desde el año 2003 (Soza-Amigo y Aroca (2011)); por su parte, las actividades y productos que más aportan para que aumenten en una unidad los insumos primarios (suma en filas de la matriz inversa de Ghosh), serían prácticamente las tres, puesto que el sector primario y terciario presentan el mismo impacto, en torno a las dos unidades; sin embargo, claro ésta, que el sector que más motiva cambios en los insumos primarios es el industrial.

\section{IMPORTANCIA DE LOS SECTORES}

En la Fig. 1 se muestra la importancia relativa de los sectores en el producto interno bruto de la comuna de Punta Arenas para el año 2003, año base de nuestra comparación.

Doce de los cuarenta sectores considerados producen más del 90 por ciento del PIB y más de 41 por ciento de él, es producido por el sector minero y de productos químicos, es decir, la industria del petróleo y el metanol.

Un segundo grupo, que podríamos caracterizar como el sector de los servicios (privados) aporta más del 25 por ciento del PIB de la comuna y el sector de servicios de administración pública tiene un aporte de 13 por ciento, lo que lo hace un sector importante en Punta Arenas.

\section{LOS CAMBIOS DEL QUINQUENIO}

En el quinquenio que va desde 2003 a 2007, se aprecian algunos cambios significativos en estos sectores. El sector que destaca en términos positivo en el de servicios empresariales con un crecimiento de más del 100\%, mientras que la construcción y los servicios inmobiliarios y de propiedad de vivienda son los que mas caen, con más de 41 por ciento cada uno. Los resultados para los doce principales sectores de la economía, se presentan a continuación (Fig. 2). 


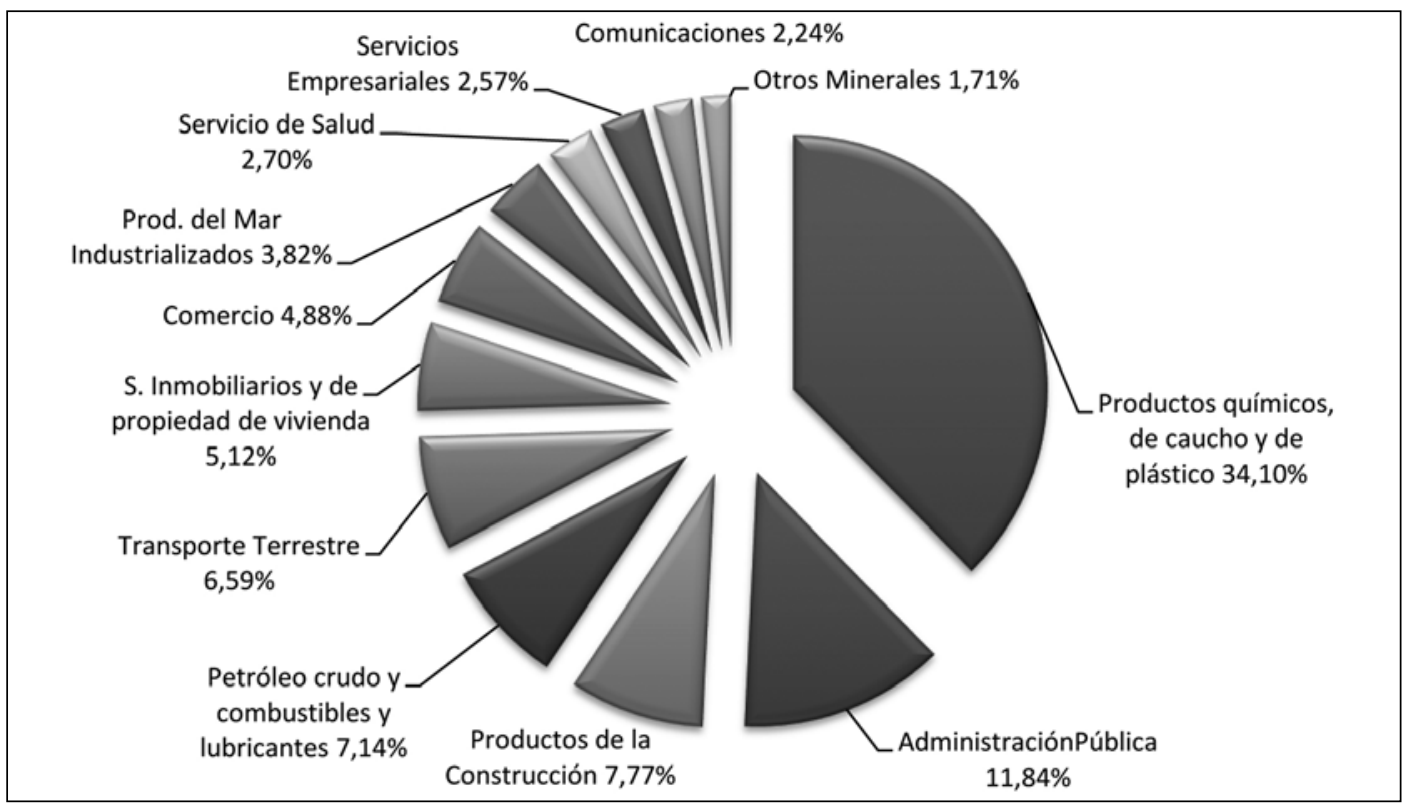

Fig. 1. Participación principales sectores en el PIB del año 2003. Fuente: Propia.

Otra tendencia que emerge claramente de estos cambios, es que los servicios en general, pero especialmente los empresariales, de comunicaciones y comerciales son los que más crecen en el periodo de análisis, los cuales representan en conjunto un 10 por ciento de la economía, mientras que sectores importantes, como los asociados a la explotación y manufactura de minerales caen significativamente, como los son el petróleo crudo, combustibles y lubricantes y el de productos químicos, de caucho y plásticos, que en promedio reducen su aporte al PIB comunal en un 14 por ciento. Estos dos sectores representan más del 41 por ciento de la economía comunal, por lo que su

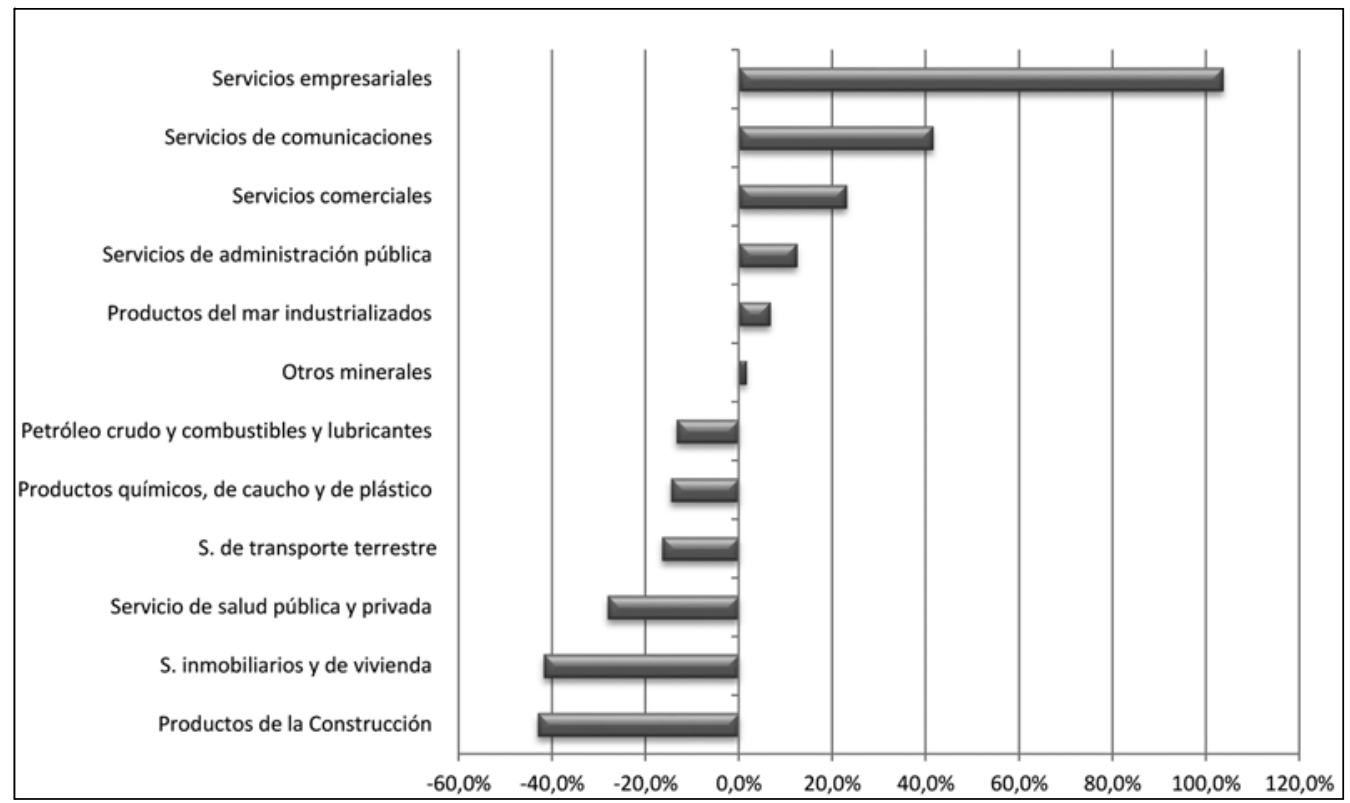

Fig. 2: Variaciones del PIB por sector entre 2003 y 2007. Fuente: Propia. 
caída en estos términos tiene implicancias significativas en el desarrollo económico de la zona y efectos multiplicadores que expanden los impactos negativos de esta reducción, que se ve aplacada por lo que pasa en los sectores servicios de la región, que aunque de menor magnitud, presentan crecimientos significativos.

\section{LOS IMPACTOS DE LOS CAMBIOS EN EL PIB SOBRE LA PRODUCCIÓN TOTAL}

Los cambios ocurridos entre 2003 y 2007 en los principales sectores de la comuna de Punta Arenas relacionados con el producto se pueden apreciar en dos dimensiones. La primera dice relación con los encadenamientos de los sectores con la economía comunal. El gráfico de la Fig. 3 muestra que existe un incremento generalizado de estos.

A diferencia de los cambios producidos en los niveles de producción, los encadenamientos en general muestran incrementos muy significativos, especialmente en el sector de servicios ofrecidos por los sectores privados como los servicios comerciales, de comunicaciones, de transporte y empresariales. Al mismo tiempo, también ocurren incrementos significativos en los sectores asociados a la producción y elaboración basada en materias primas como Otros Minerales, Petróleo y Productos Químicos, al igual que el sector de la Construcción y de elaboración de Productos del Mar.

Los sectores asociados a la administración tienden a mantener su nivel de encadenamiento (relaciones de compra y venta de insumos) con los demás sectores de la economía local al igual que el sector de servicios inmobiliarios.

Este proceso es interesante, ya que refleja un proceso de creciente interacción o de aumento de los encadenamientos, lo que implica una economía mas compleja, alejándose de los enclaves productivos y dando muestra de un mayor desarrollo.

Sin embargo, el efecto positivo de este crecimiento en los encadenamientos productivos se ha visto disminuido por la reducción en el producto interno bruto, que es equivalente a una baja en la demanda final por bienes y servicios en la comuna de Punta Arenas entre los años 2003 a 2007.

Por lo tanto un incremento en los encadenamientos productivos amplifica el impacto de las variaciones en la demanda final o PIB sobre la comuna, como lo muestra el siguiente gráfico (Fig. 4).

Como se puede apreciar, los servicios empresariales y comerciales que tienen una variación positiva en el periodo de 22 y 9 mil millones de pesos respectivamente, generan un efecto positivo conjunto de casi 52 mil millones de pesos sobre la producción de la economía de Punta Arenas. Sin

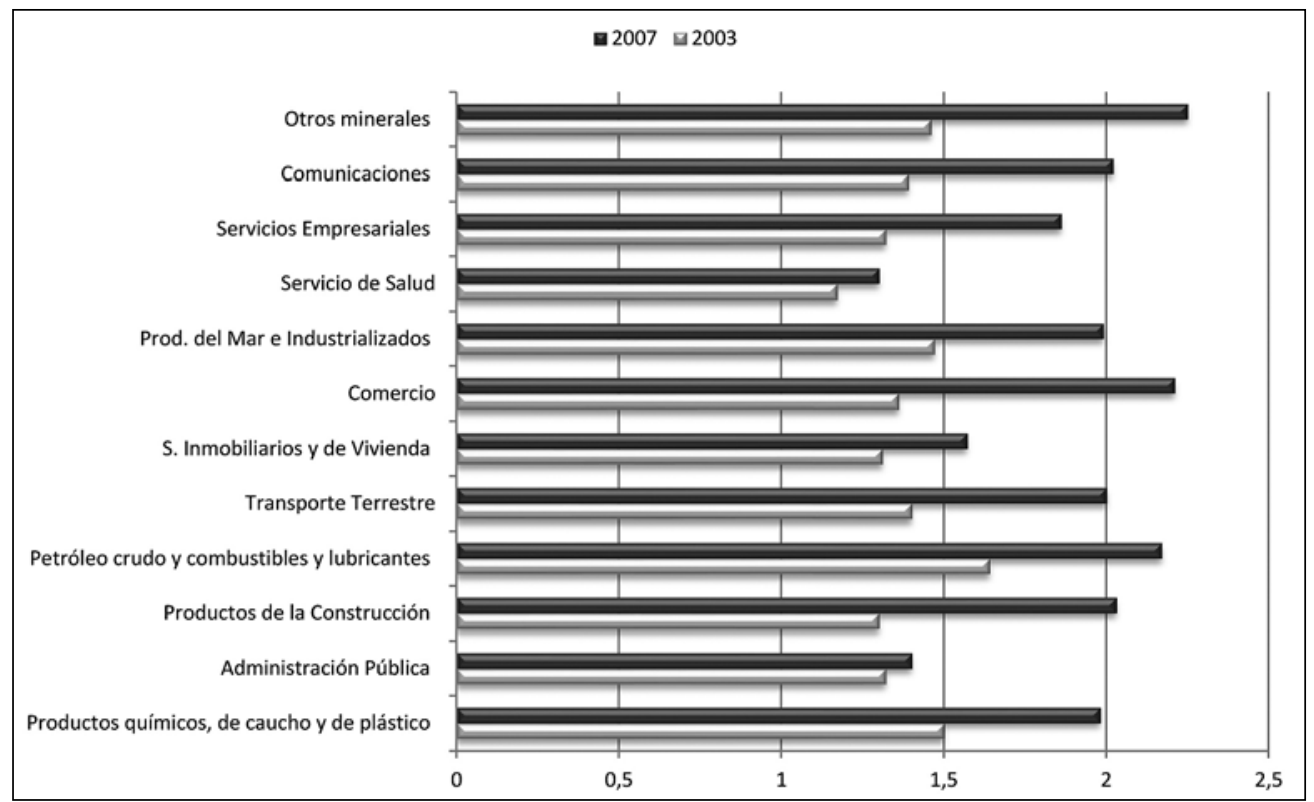

Fig. 3. Multiplicadores de producto para los principales sectores de la comuna de Punta Arenas. Fuente: Propia. 


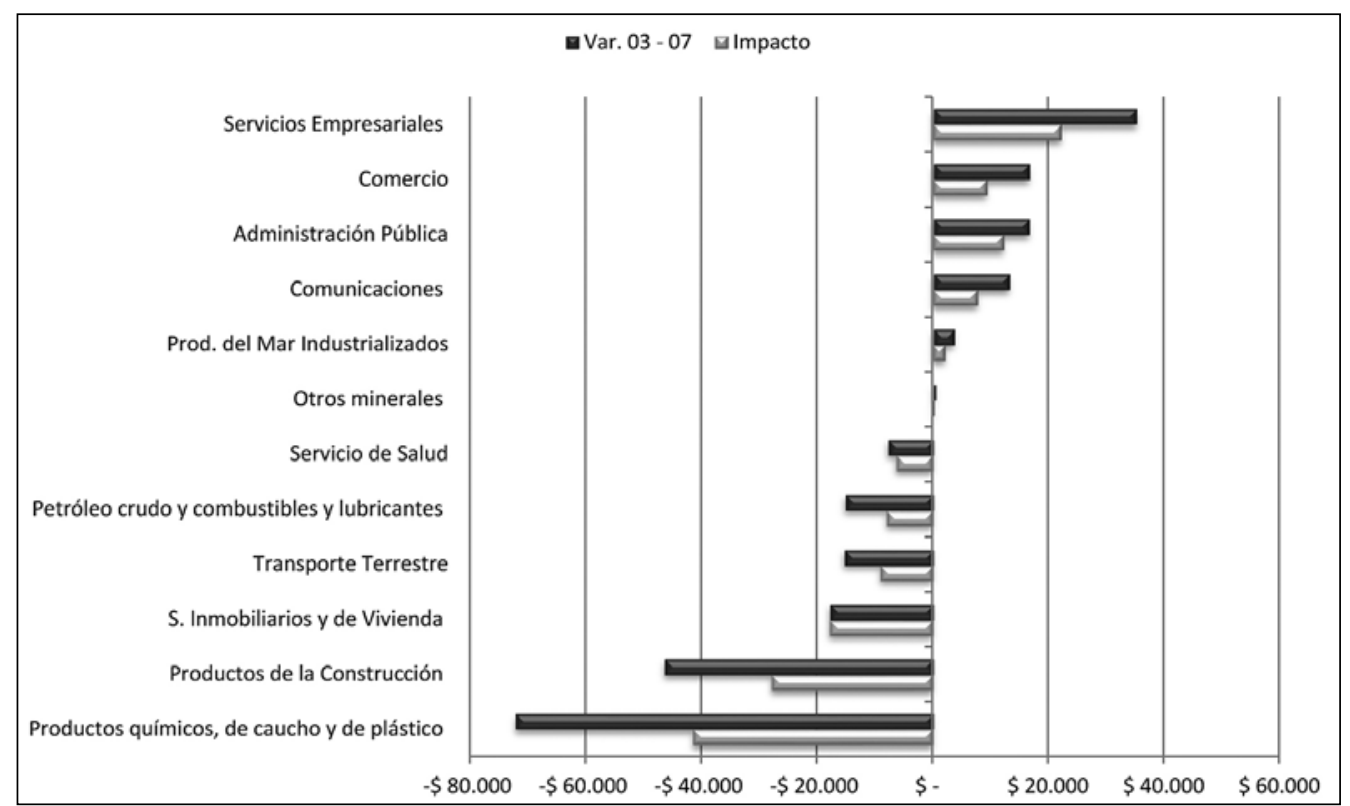

Fig. 4. Variación en el PIB e impacto en el producto comunal. Fuente: Propia.

embargo, la caída del sector de productos químicos (metanol) de 41 millones de pesos, genera un impacto negativo en la producción comunal de más de 72 mil millones, muy superior al impacto positivo de los dos sectores mencionados previamente.

Cuando se calcula el efecto total neto de los cambios ocurridos en la demanda final o PIB comunal, en el quinquenio, se obtiene una reducción en el valor de la producción de más de 96 mil millones de pesos, que son cercanos a los 200 millones de dólares. Para tener una idea de esta reducción en el valor bruto de la producción de bienes y servicios de la comuna de Punta Arenas, la región de Magallanes recibió como aporte total durante los 5 años analizados por concepto de Fondo Nacional de Desarrollo Regional menos de 150 millones de dólares, es decir, en promedio alrededor de 30 millones de dólares por año; situación similar para los casos que tratan se observa en Aroca (2001); Aroca y Atienza (2008) y, en Soza-Amigo y Aroca (2010).

\section{LOS IMPACTOS DE LOS CAMBIOS EN LA DEMANDA FINAL O PIB SOBRE EL INGRESO}

Para investigar sobre el impacto en los ingresos, cuya importancia al desarrollo se plantea por Isard et al (1998), especialmente en las remuneraciones de los habitantes de la comuna de Punta Arenas se estimaron los multiplicadores sectoriales del ingreso. Estos miden la cantidad o proporción del cambio en la demanda final que se traducirá en ingreso para los trabajadores de la zona.

En estricto rigor, se define como el incremento en las remuneraciones que ocurrirá por el incremento en una unidad de demanda final o producto interno bruto. El gráfico de la Fig. 5 muestra los multiplicadores de ingreso para los años 2003 y 2007, revelando también los cambios ocurridos en ellos.

Este indicador muestra por ejemplo que por cada unidad o millón de pesos producido por los Servicios de Administración Pública en el 2003, se destinaban a remuneraciones 720 mil pesos, uno de los más altos del grupo analizado. Mientras que esta misma relación en el 2007 para el sector de Productos Químicos (metanol) implicaba que por cada millón de pesos exportados o vendidos, se destinaba a pagar remuneraciones la cantidad de 250 mil pesos, uno de los más bajos entre el grupo de sectores estudiado.

Casi todos los sectores presentan un incremento en su multiplicador de ingreso, excepto por los Servicios de Administración Pública que se reduce y el de Servicios de Salud que se mantiene constante. Mientras que el primero refleja el efecto del Estado, que de acuerdo a estos datos, estaría invirtiendo más capital, lo que reduce la participación relativa de los trabajadores en sus procesos productivos. 


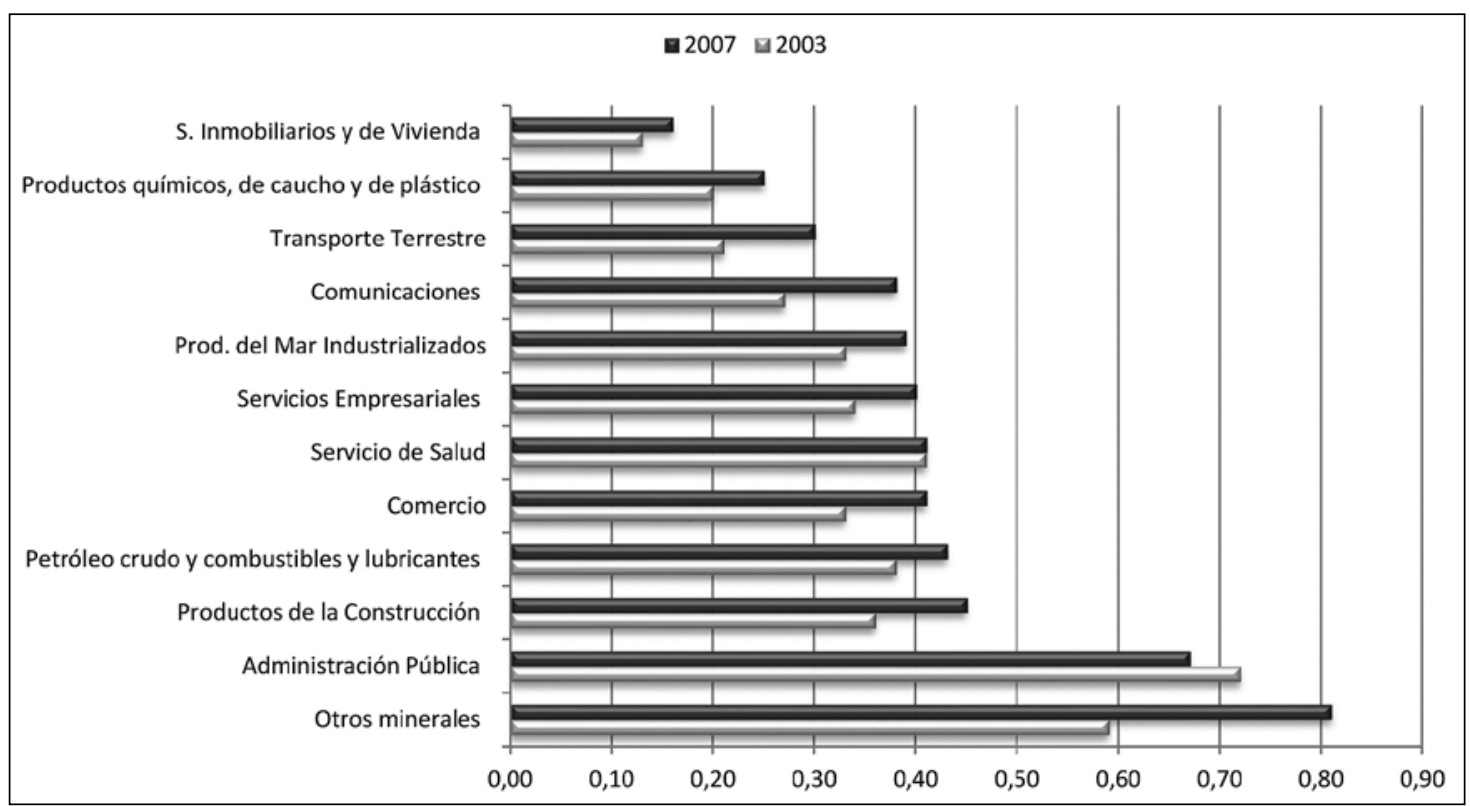

Fig. 5. Multiplicadores de ingreso de la comuna de Punta Arenas. Fuente: Propia.

El resto de los sectores presenta un incremento, aunque no tan significativo como lo ocurrido con los multiplicadores de producto. Este resultado es interesante, ya que el ingreso obtenido a través de las remuneraciones generalmente en un mejor indicador del bienestar de la población que el Producto Interno Bruto realizado en la comuna. Es decir, el incremento en producción por encadenamientos productivos es superior en el periodo al incremento en multiplicadores de ingreso, y como consecuencia en el bienestar de su comunidad, lo que es un patrón que se repite sistemáticamente en economía dominadas por sectores asociados a materias primas como es el caso del petróleo y metanol en Punta Arenas.

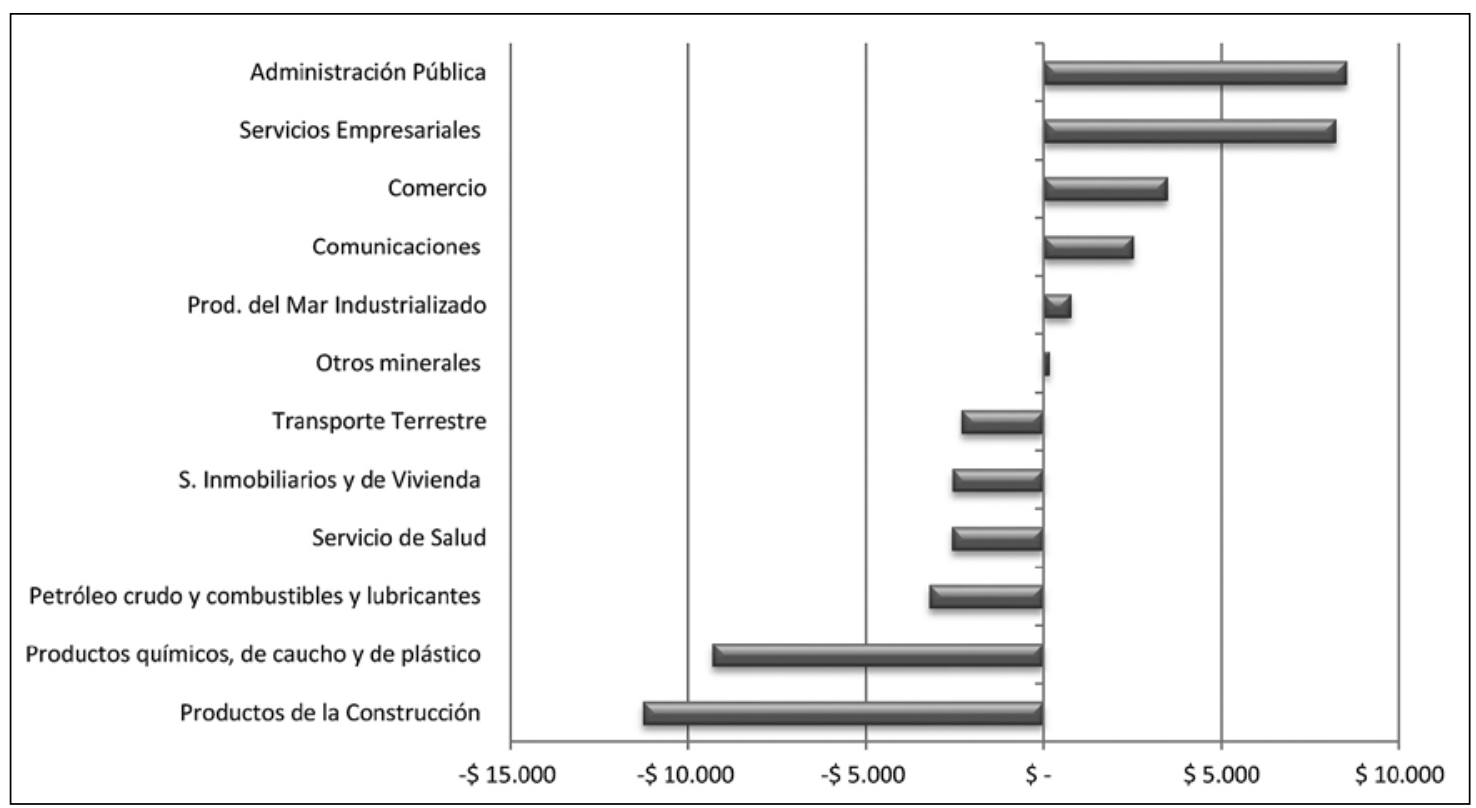

Fig. 6. Impacto del cambio en el PIB entre 2003 y 2007 sobre el Ingreso (en millones de pesos). Fuente: Propia. 
Los cambios más negativos en las remuneraciones pagadas, están asociados a los sectores de Productos Químicos y de la Construcción, mientras que los servicios tantos privados como públicos son los privados Empresariales, Comerciales y de Comunicaciones son los que explican la mayor parte del aumento en el pago de remuneraciones en la comuna.

Cuando se suman todos los impactos sectoriales sobre los pagos en remuneraciones, el saldo netos es dominado por lo que ocurre en la Construcción y los productos Químicos, teniendo un cambio neto negativo superior a los 7.700 millones de pesos.

\section{CONCLUSIONES}

La estimación de las matrices de insumo producto para la comuna de Punta Arenas para los años 2003 y 2007 ha sido un aporte que permite entender la historia de los cambios económicos ocurridos en el quinquenio.

En este trabajo se intento construir parte de esa historia, detallando que la economía de la comuna de Punta Arenas sigue dominada por la producción asociada a sus principales materias primas: petróleo y metanol. Sin embargo, comienza a aparecer una interesante tendencia donde los sectores de servicios comienzan a tener mayor importancia, entre ellos el de servicios empresariales. Aquí surge una primera línea de investigación futura, este incremento significativo en este sector se debe a un cambio en la estrategia de subcontratación de las empresas asociadas a materias primas o es un cambio genuino en el sector; en tal sentido, habrá que preguntarse si en 2007 existía un conjunto importante de empresas que dan servicios empresariales que han hecho que la comuna tenga una economía más compleja, desafortunadamente las tablas de insumo-producto no permiten responder esta interrogante, por lo que quedará como desafío para una futura investigación.

Un segundo conjunto de conclusiones tiene que ver con el impacto de los cambios ocurridos en el quinquenio. Primero, se aprecia una reducción general del tamaño de la economía explicada principalmente por la caída en la producción de los sectores asociadas a las materias primas y este impacto negativo ha sido atenuado por el crecimiento de los sectores de servicios, que se han convertido en el otro motor de la comuna de Punta Arenas.
También se pudo constatar que los cambios significativos ocurridos en la producción no se tradujeron con igual intensidad en cambios en remuneraciones para los habitantes de la región. Implicando que el impacto del crecimiento sobre el bienestar de los habitantes será más lento, lo cual es una características de economías basadas en recursos naturales, por lo que la tendencia en el crecimiento del sector servicios puede ser una fuerza que puede cambiar este hecho a futuro, lo que hace recomendable su fortalecimiento, especialmente mirando hacia actividades complementarias a las productivas basadas en materias primas como pueden ser las asociadas al sector turismo.

\section{BIBLIOGRAFÍA}

AROCA, P. 2001. Impacts and Development in Local Economies Based on Mining: The case of the Chilien II Region. Resources Policy 27:119-134.

AROCA, P. y M. ATIENZA. 2008. La Conmutación Regional en Chile y su Impacto en la Región de Antofagasta. Revista EURE XXXIV(102):97-120.

ISARD, W., I. AZIS, M. DRENNAN, S. SALTZMAN y E. THORBECKE. 1998. Methods of Interregional and Regional Analysis, Ashgate, Hants.

SOZA-AMIGO, S. 2008. Relaciones Intersectoriales en Magallanes: En busca de su estructura económica. Magallania 36(1):79-102.

2009. Análisis Comparativo para la Economía Magallánica Desde la Perspectiva de la Sensibilidad de Coeficientes Técnicos. Magallania 37(1):133-151.

2010. Matriz Insumo Producto para la Comuna de Punta Arenas: Obtención y Análisis de Resultados. Ediciones Universidad de Magallanes, Punta Arenas.

SOZA-AMIGO, S. y P. AROCA. 2010. Oportunidades Pérdidas en Magallanes. Magallania 38(2): 61-73.

2011. Small, Isolated and Commodities Dependent Economies: The case of the Oil and Methanol in the County of Punta Arenas, Chile. Magallania, 39(2):113135.

SOZA-AMIGO, S. y C. RAMOS. 2011 La Agregación en las Tablas Insumo-producto: Una revisión desde la perspectiva de las ramas que no se unen. Revista de Economía Mundial 28: 247-276.

SOZA-AMIGO, S. y J. MARIPANI. 2012. Cambios Estructurales y Efectos del Turismo en Punta Arenas: Análisis de las matrices Input-Output para los Años 2007 y 2003. Universidad de Magallanes, Punta Arenas (en prensa). 


\section{ANEXO 1}

Formulaciones que determinan el tipo de igualdades para una TIO y encadenamiento según las distintas técnicas empleadas (hacia atrás (BL) y delante (FL) $)^{5}$

Ventas ínterindustriales $\left(z_{\mathrm{ij}}\right)+$ demanda final $\left(\mathrm{c}_{\mathrm{i}}\right.$; consumo, ofd; resto de demanda final $)=$ ventas totales $\left(\mathrm{x}_{\mathrm{i}}\right)$

$\sum_{j=1}^{n} z_{i j}+c_{i}+o f d_{i}=x_{i}$

Compras interindustriales $\left(\mathrm{z}_{\mathrm{ij}}\right)+$ valor agregado $\left(\mathrm{w}_{\mathrm{j}}\right.$ : salarios; $\mathrm{ov}_{\mathrm{j}}$ : resto del valor agregado)+ importaciones $\left(\mathrm{m}_{\mathrm{j}}\right)=$ salidas totales $\left(\mathrm{x}_{\mathrm{j}}\right)$

$$
\sum_{j=1}^{n} z_{i j}+w_{j}+o v_{j}+m_{j}=x_{j}
$$

Matriz de Coeficientes Técnicos Matriz de Coeficientes de Distribución

$$
\mathbf{A}=\mathbf{a}_{\mathrm{ij}}=\frac{\mathbf{Z}_{\mathrm{ij}}}{\mathbf{x}_{\mathrm{j}}}=\begin{array}{cccc}
\mathbf{a}_{11} & \mathbf{a}_{12} & \cdots & \mathbf{a}_{1 \mathrm{n}} \\
\vdots & \vdots & \ddots & \vdots \\
\mathbf{a}_{\mathrm{n} 1} & \mathbf{a}_{\mathrm{n} 2} & \cdots & \mathbf{a}_{\mathrm{nn}}
\end{array} \quad \overrightarrow{\mathbf{A}}=\overrightarrow{\mathbf{a}_{\mathrm{ij}}}=\frac{\mathbf{Z}_{\mathrm{ij}}}{\mathbf{x}_{\mathbf{i}}}=\begin{array}{ccccc}
\overrightarrow{\mathbf{a}}_{11} & \overrightarrow{\mathbf{a}}_{12} & \cdots & \overrightarrow{\mathbf{a}}_{1 \mathrm{n}} \\
\overrightarrow{\mathbf{a}}_{\mathrm{n} 1} & \overrightarrow{\mathbf{a}_{\mathrm{n} 2}} & \cdots & \overrightarrow{\mathbf{a}}_{\mathrm{nn}}
\end{array}
$$

Matriz Inversa de Leontief Matriz Inversa de Ghosh

$$
(\mathbf{I}-\mathbf{A})^{-1}=\left[\begin{array}{lcccc}
\mathbf{b}_{\mathrm{ij}} & \mathbf{b}_{11} & \mathbf{b}_{12} & \cdots & \mathbf{b}_{1 \mathrm{n}} \\
\vdots & \vdots & \ddots & \vdots \\
\mathbf{b}_{\mathrm{n} 1} & \mathbf{b}_{\mathrm{n} 2} & \cdots & \mathbf{b}_{\mathrm{nn}}
\end{array} \quad \overrightarrow{\mathbf{B}}=(\mathbf{I}-\overrightarrow{\mathbf{A}})^{-1}=\left[\overrightarrow{\mathbf{b}}_{\mathrm{ij}}\right]=\begin{array}{ccccc}
\overrightarrow{\mathbf{b}}_{11} & \overrightarrow{\mathbf{b}}_{12} & \cdots & \overrightarrow{\mathbf{b}}_{1 \mathrm{n}} \\
\vdots & \vdots & \ddots & \vdots \\
& \overrightarrow{\mathbf{b}}_{\mathrm{n} 1} & \overrightarrow{\mathbf{b}}_{\mathrm{n} 2} & \cdots & \overrightarrow{\mathbf{b}}_{\mathrm{nn}}
\end{array}\right.
$$

Multiplicador de Producción Multiplicador de Ingreso
$\mathbf{O}_{\mathrm{j}}=\sum_{i=1}^{n} \mathbf{b}_{\mathrm{ij}}$
$\mathbf{H}_{\mathrm{j}}=\sum_{\mathrm{i}=1}^{\mathrm{n}} \frac{\mathbf{w}_{\mathrm{i}}}{\mathbf{x}_{\mathrm{i}}} \mathbf{b}_{\mathrm{ij}}$ 


\section{ANEXO 2 \\ Obtención e interpretación de matrices de coeficientes de distribución e inversas ${ }^{6}$}

Cada elemento de la matriz de coeficientes de distribución se designa genéricamente como $\left(\overrightarrow{\mathbf{a}}_{\mathrm{ij}}\right)$ y se calcula como $z_{\mathrm{i}} / \mathrm{x}_{\mathrm{i}}$, donde $\mathrm{x}_{\mathrm{i}}$ es el output de la rama i-ésima. Por lo tanto, cada coeficiente de la matriz de distribución, mostrará la proporción en términos monetarios que emplea la rama de la fila i-ésima, y que se destina a cada una de las otras ramas o a la demanda final. Con esta nueva forma de plantear el problema, se da paso a que los insumos primarios (trabajo o capital empleado en la producción de esa j-ésima rama) sean las nuevas variables exógenas y no la demanda final, como en el caso de la matriz de coeficientes técnicos. De esta forma, los modelos de demanda (Leontief) y oferta (Ghosh) permitirían conocer cómo los cambios exógenos que de allí se puedan plantear afectan a las distintas funciones de producción y distribución, ya que ahora se puede trabajar con dos variables exógenas (demanda final $e$ input primarios), lo que le daría a los usos que se pueden obtener de las tablas mayor flexibilidad e interpretación.

Por otro lado y, aún cuando, la información que se deriva de una matriz de coeficientes técnicos o de distribución es muy importante, dado que presenta una idea clara y precisa de cuáles son los requerimientos directos y su distribución en el entramado económico que realiza cada actividad para su proceso productivo, lo es más, el saber cuáles son también los requerimientos indirectos; esto es, también interesa indagar, cómo una determinada actividad afecta al resto, no sólo en términos directos sino que también indirectos. La información a que se hace mención, se puede obtener a partir de las matrices inversas de Leontief y de Ghosh.

La operatoria que da origen a estas matrices es equivalente a sumar y multiplicar ad infinitum la matriz de coeficientes técnicos o la de distribución según sea el caso $\left(A+A^{2}+A^{3}+A^{4}+\ldots A^{n} ; o, B+B^{2}+\right.$ $\left.\mathrm{B}^{3}+\mathrm{B}^{4}+\ldots \mathrm{B}^{\mathrm{n}}\right)$, como se puede apreciar y luego de plantear que tal cambio obedece a una variación en la demanda final (o, input primario si se empleo la matriz de distribución), se originan una serie de pasos en términos de efectos multiplicativos, lo anterior luego de simplificaciones y arreglos dan lugar a la expresión matemática que se conoce como la inversa de Leontief o la de Ghosh cuando corresponde ${ }^{7}$.

Cada elemento de la matriz inversa de Leontief $\left(\mathrm{x}=\mathrm{B}=(\mathrm{I}-\mathrm{A})^{-1}\right.$, con $\mathrm{b}_{\mathrm{ij}} \mathrm{I} \mathrm{B}$, donde $\mathrm{x}$ es la producción, $\mathrm{I}$ una matriz identidad y $\mathrm{A}$, la matriz de coeficientes técnicos), expresará en cuanto se debe incrementar la producción de la rama i-ésima si se desea que aumente en una unidad la demanda final de la j-ésima. Por su parte, su suma en columnas, debe ser entendida como el aumento de la producción que debe realizar todo el sistema económico, a fin de satisfacer el requerimiento de una unidad por parte de la j-ésima rama (Rasmussen, pp. 127). Asimismo, la suma de una fila de la matriz inversa de Leontief, puede ser entendida como el aumento en la producción que debe realizar la i-ésima rama, cuando cada rama del sistema aumenta simultáneamente en una unidad su demanda final (Rasmussen, pp. 127).

6 Extraído y adaptado de Análisis de Impacto para la MIPPA. En: Matriz Insumo-Producto para la Comuna de Punta Arenas: Obtención y Análisis de Resultados, por Sergio Soza-Amigo, pp. 107, Ed. Universidad de Magallanes, 2010.

7 Para un mayor detalle y comprensión de estas matrices puede leerse Goodwin (1949) o, Soza-Amigo (2007). 


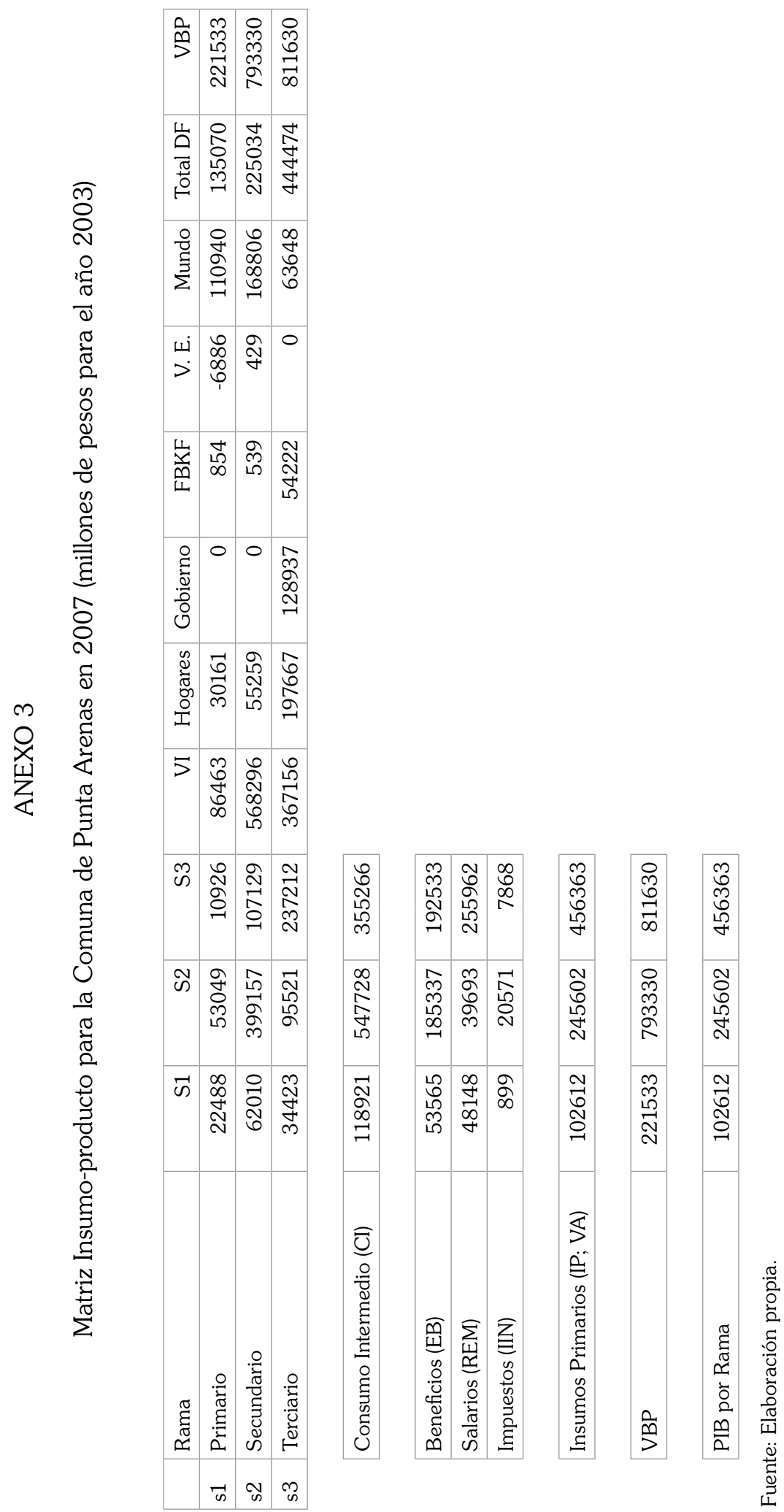


ANEXO 4

Matriz de Coeficientes Técnicos para la Comuna de Punta Arenas (2007)

\begin{tabular}{|c|c|c|c|c|}
\hline & Rama & s1 & s2 & s3 \\
\hline s1 & Sector Primario & 0,1015 & 0,0669 & 0,0135 \\
\hline s2 & Sector Secundario & 0,2799 & 0,5031 & 0,1320 \\
\hline s3 & Sector terciario & 0,1554 & 0,1204 & 0,2923 \\
\hline & Consumo Intermedio (CI) & 0,5368 & 0,6904 & 0,4377 \\
\hline & Beneficios y Excedente Bruto de Explotación & 0,2418 & 0,2336 & 0,2372 \\
\hline & Salarios y Remuneraciones (REM) & 0,2173 & 0,0500 & 0,3154 \\
\hline & Impuestos (IIN) & 0,0041 & 0,0259 & 0,0097 \\
\hline & Insumos Primarios (IP; VA) & 0,4632 & 0,3096 & 0,5623 \\
\hline & Valor Bruto de la Producción (VBP) & 1,0000 & 1,0000 & 1,0000 \\
\hline
\end{tabular}

Fuente: Elaboración propia.

ANEXO 5

Matriz de Coeficientes de Distribución para la Comuna de Punta Arenas (2007)

\begin{tabular}{|r|l|r|r|r|r|r|r|}
\hline & Rama & s1 & s2 & s3 & VI & Total DF & VBP \\
\hline s1 & Sector Primario & 0,1015 & 0,2395 & 0,0493 & 0,3903 & 0,6097 & 1,0000 \\
\hline s2 & Sector Secundario & 0,0782 & 0,5031 & 0,1350 & 0,7163 & 0,2837 & 1,0000 \\
\hline s3 & Sector terciario & 0,0424 & 0,1177 & 0,2923 & 0,4524 & 0,5476 & 1,0000 \\
\hline
\end{tabular}

Fuente: Elaboración propia.

ANEXO 6

Matriz Inversa de Leontief (2007)

\begin{tabular}{|r|l|r|r|r|r|}
\hline & Rama & s1 & s2 & s3 & Suma \\
\hline s1 & Sector Primario & 1,1758 & 0,1714 & 0,0543 & 1,4015 \\
\hline s2 & Sector Secundario & 0,7656 & 2,2195 & 0,4285 & 3,4136 \\
\hline s3 & Sector terciario & 0,3884 & 0,4152 & 1,4978 & 2,3014 \\
\hline & Suma & 2,3297 & 2,8062 & 1,9806 & \\
\hline
\end{tabular}

Fuente: Elaboración propia.

ANEXO 7

Matriz Inversa de Distribución o Ghosh para la Comuna de Punta Arenas (2007)

\begin{tabular}{|r|l|r|r|r|r|}
\hline & Rama & s1 & s2 & s3 & Suma \\
\hline s1 & Sector Primario & 1,1758 & 0,6138 & 0,1991 & 1,9887 \\
\hline s2 & Sector Secundario & 0,2138 & 2,2195 & 0,4384 & 2,8717 \\
\hline s3 & Sector terciario & 0,1060 & 0,4059 & 1,4978 & 2,0097 \\
\hline \multirow{2}{*}{} & Suma & 1,4956 & 3,2392 & 2,1352 \\
\cline { 2 - 6 }
\end{tabular}

Fuente: Elaboración propia. 


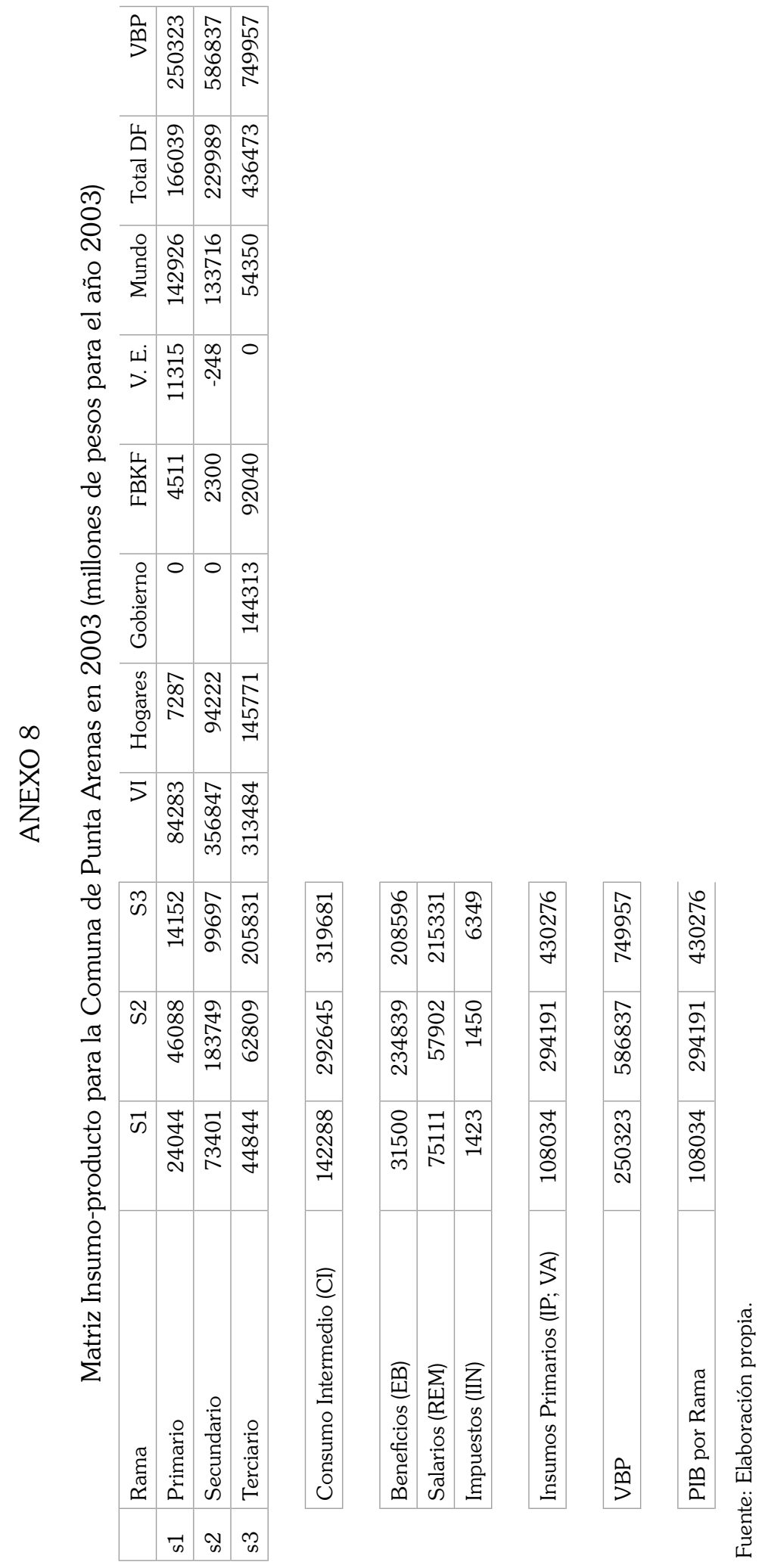




\section{ANEXO 9}

Matriz de Coeficientes Técnicos para la Comuna de Punta Arenas (2003)

\begin{tabular}{|}
\begin{tabular}{r|l|r|r|r|}
\hline s1 & Rama & sector Primario & s2 & s3 \\
\hline s2 & Sector Secundario & 0,0961 & 0,0785 & 0,0189 \\
\hline s3 & Sector terciario & 0,2932 & 0,3131 & 0,1329 \\
\hline
\end{tabular} \\
\begin{tabular}{|l|r|r|r|}
\hline Consumo Intermedio (CI) & 0,1791 & 0,1070 & 0,2745 \\
\hline Beneficios y Excedente Bruto de Explotación & 0,5684 & 0,4987 & 0,4263 \\
\hline Salarios y Remuneraciones (REM) & 0,1258 & 0,4002 & 0,2781 \\
\hline Impuestos (IIN) & 0,3001 & 0,0987 & 0,2871 \\
\hline
\end{tabular} \\
\begin{tabular}{|l|r|r|r|}
\hline Insumos Primarios (IP; VA) \\
\hline
\end{tabular} \\
\begin{tabular}{|l|r|r|r|}
\hline Valor Bruto de la Producción (VBP) & 0,0057 & 0,0025 & 0,0085 \\
\hline
\end{tabular}
\end{tabular}

Fuente: Elaboración propia.

ANEXO 10

Matriz de Coeficientes de Distribución para la Comuna de Punta Arenas (2003)

\begin{tabular}{|l|l|r|r|r|r|r|r|}
\hline & Rama & s1 & s2 & s3 & VI & Total DF & VBP \\
\hline s1 & Sector Primario & 0,0961 & 0,1841 & 0,0565 & 0,3367 & 0,6633 & 1,0000 \\
\hline s2 & Sector Secundario & 0,1251 & 0,3131 & 0,1699 & 0,6081 & 0,3919 & 1,0000 \\
\hline s3 & Sector terciario & 0,0598 & 0,0837 & 0,2745 & 0,4180 & 0,5820 & 1,0000 \\
\hline
\end{tabular}

Fuente: Elaboración propia.

\section{ANEXO 11}

Matriz Inversa de Leontief (2003)

\begin{tabular}{|l|l|r|r|r|r|}
\hline & Rama & s1 & s2 & s3 & Suma \\
\hline s1 & Sector Primario & 1,1634 & 0,1418 & 0,0562 & 1,3614 \\
\hline s2 & Sector Secundario & 0,5685 & 1,5679 & 0,3021 & 2,4385 \\
\hline s3 & Sector terciario & 0,3711 & 0,2663 & 1,4367 & 2,0741 \\
\hline & Suma & 2,1030 & 1,9760 & 1,7950 &
\end{tabular}

Fuente: Elaboración propia.

ANEXO 12

Matriz Inversa de Distribución o Ghosh para la Comuna de Punta Arenas (2003).

\begin{tabular}{|l|l|r|r|r|r|}
\hline & Rama & s1 & s2 & s3 & Suma \\
\hline s1 & Sector Primario & 1,1634 & 0,3324 & 0,1685 & 1,6643 \\
\hline s2 & Sector Secundario & 0,2425 & 1,5679 & 0,3860 & 2,1964 \\
\hline s3 & Sector terciario & 0,1239 & 0,2084 & 1,4367 & 1,7690 \\
\hline
\end{tabular}

Fuente: Elaboración propia. 
